\title{
ERRATUM
}

\section{Health-related quality of life in persons with long-standing spinal cord injury}

IB Lidal, M Veenstra, N Hjeltnes and F Biering-Sørensen

Spinal Cord (2008) 46, 765; doi:10.1038/sc.2008.41

Correction to: Spinal Cord (2008) advance online publication, 11 March 2008; doi:10.1038/sc.2008.17

Since the publication of the above article, the authors have noticed that the placement of the words 'social functioning' is incorrect under the Methods and Results heading within the abstract section.

The correct placement of the words 'social functioning' is shown below.

Methods: Survey data and clinical examination of 165 persons with traumatic SCI of more than 20 years duration.
HRQOL was assessed with the Norwegian 36-item short-form (SF-36) Health Survey. The SF-36 results were compared with Norwegian norm data adjusted to age and gender.

Differences in HRQOL between subgroups were studied.

Results: The persons with SCI exhibited significantly decreased HRQOL in the subscales for Physical Functioning, Bodily Pain, General Health, Social Functioning and Vitality compared to the normal population.

There...

The typesetters would like to apologize for this mistake. 